Dominika Romanowska

\title{
TRADUZIONI NEL CAMPO DI ASSICURAZIONI SOCIALI
}

\author{
Translations in the area of social insurance scheme
}

\begin{abstract}
The subject of the present article is the translation between Polish and Italian social security systems. In the first part the reader shall be informed about how each system has been formed throughout the history. Different formation of systems resulted in different structures of the institutions in Poland and in Italy. The second part briefly presents those structures - Polish ZUS and Italian INPS and INAIL - and lists the responsibilities of each of them which helps to spot the differences. The ultimate, essential part, demonstrates difficulties faced by translators specialized in the field of social insurance. Several terms represented in one language can have various formulations in the other one according to the context in which they occur. There are also terms that have nonequivalent in the other system. The most common ones are listed in the chart in order to present every possible translation of the potentially problematic lemmas.
\end{abstract}

Key words: Italian-Polish legal translation, Italy's social insurance scheme, nonequivalent terms, Polish pension system, specialized translation

Parole chiave: sistema di previdenza sociale italiano, sistema pensionistico polacco, termini non-equivalenti, traduzione legale italo-polacca, traduzione specializzata

Słowa kluczowe: polski system emerytalny, terminy bezekwiwalentowe, tłumaczenia specjalistyczne, włoski system ubezpieczeń społecznych, włosko-polski przekład prawniczy

Nel presente articolo vorrei discutere il tema delle difficoltà nella traduzione nel campo della previdenza sociale dal polacco in italiano e dall'italiano in polacco.

Un noto economista tedesco, Johann-Matthias Graf von der Schulenburg,

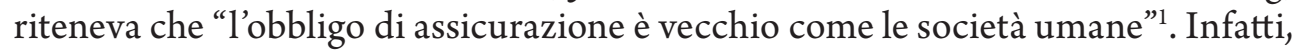

1 J. M. Graf von der Schulenburg, Pflichtversicherung - ein historischer Rückblick, [in:] Pflichtversicherung. Segnung oder Sündenfall. Dokumentation über ein Symposium am 28-30 Oktober 2004 im Schloss Marbach, Öhningen, Karlsruhe 2005, p. 21. In: M. Orlicki, Ubezpieczenia obowiązkowe, Wolters Kluwer, Warszawa 2011, p. 26. 
si può dire che le assicurazioni esistevano in varie forme in tempi diversi sin da quando esiste l'uomo. L'uomo come un essere sociale cerca di creare legami sociali, di far parte di una comunità, di ricercare nel gruppo sicurezza e stabilità. Le società umane fornivano da sempre assistenza ai propri membri in stato di necessità. Le assicurazioni per le imprese esistevano dunque sin dall'antichità.

Il potenziamento e l'espansione delle assicurazioni sociali moderne sono dovute alla rivoluzione industriale del diciottesimo secolo. Durante quel periodo il numero della gente che si manteneva lavorando nell'industria è aumentato significamente. Eseguire lavoro in tale settore presentava rischi di malattie e infortuni, i quali in seguito potevano rendere un lavoratore privo di mezzi di sostentamento. Nello stesso tempo si poteva notare che i legami familiari si allentavano, fra l'altro a causa della migrazione dalle zone rurali verso le grandi città, e sempre più persone anziane e innabili al lavoro non potevano contare sull'aiuto da parte dei membri della famiglia che in molti casi reggevano sulle loro spalle, ovvero sia sulla sussistenza finanziaria del nucleo. Cresceva infatti la necessità di regolare in qualche maniera la responsabilità dei datori di lavoro per quanto riguarda i danni conseguenti a infortuni sul lavoro avvenuti durante la prestazione professionale in modo da proteggere i lavoratori (specialmente gli operai) contro un eventuale indigenza.

Quando nel Settecento in Inghilterra è scoppiata la rivoluzione industriale, venivano create Casse di mutuo soccorso ${ }^{2}$ (Fund of Mutual Assistance) che si sono sviluppate molto nell'Ottocento quando altri paesi hanno scoperto il loro ruolo positivo. I membri di dette Casse pagavano regolarmente un contributo e in cambio in caso di malattia o invalidità ricevevano un' indennità che garantiva mantenere il reddito. Inoltre venivano create altre forme di auito alle persone più esposte al rischio di indigenza ossia orfani, vedove e persone che a causa della loro età o per qualche altro motivo non erano capaci di lavorare. Con il sempre più veloce sviluppo dell'industria aumentava esigenza di creare un sistema comune e obbligatorio per tutti i lavoratori e datori di lavoro che avrebbe potuto assistere lavoratori in una situazione di bisogno.

Lassicurazione sociale nella forma molto vicina a quella che oggi conosciamo fu creata in Germania. Sotto l'impero di Guglielmo I negli anni 1883, 1884 e $1889^{3}$ furono varate leggi sulle assicurazioni sociali che riguardavano, rispettivamente, malattie, infortuni sul lavoro nonché invalidità, vecchiaia e morte ${ }^{4}$. Le suddette leggi furono poi codificate nel codice di previdenza sociale e questo era la prima volta a usare il termine previdenza sociale. Il sistema che fu

${ }^{2}$ http://www.storiadellasicurezzasociale.ch/fileadmin/synthese-it.pdf, p. 3 (accesso: 22.09.2017).

${ }^{3}$ http://www.zus.com.pl/files/prasa/Pocz\%C4\% 85tki\%20i\%20rozw\%C3\%B3j\%20ubezpiecze\%C5\%84.pdf (accesso: 22.09.2017).

${ }^{4}$ Ibidem. 
introdotto nella Germania dell'epoca veniva adottato in seguito nella maggior parte dei paesi europei.

Nell'Ottocento, quando la rivoluzione industriale arrivò in Italia, detto paese doveva affrontare i problemi con essa connessi. Si osservò un'esigenza di statalizzare il settore di assicurazioni per garantire aiuto di cui la gente aveva bisogno. Il primo passo al livello statale era la fondazione della Cassa Nazionale di Previdenza per l'Invalidità e la Vecchiaia degli operai (che poi fu rinominata la Cassa Nazionale delle Assicurazioni Sociali). Con quell'ente è stata istitutia la forma facoltativa di tutela pensionistica per i manodopera. Grazie a detto ente gli operai che volevano assicurarsi contro l'invalidità e la vecchiaia, ne avevano possibilità. Il secondo passo dello sviluppo di assicurazioni in Italia ebbe luogo dopo prima guerra mondiale, nel 1919. In quell'anno Istituto fu denominato la Cassa Nazionale delle Assicurazioni Sociali e le assicurazioni diventarono obbligatorie. Nel 1933 sotto il regime fascista nacque INFAIL - Istituto Nazionale Fascista per l'Assicurazione contro gli Infortuni sul Lavoro e la Cassa Nazionale assunse la denominazione di Istituto Nazionale Fascista della Previdenza Sociale. Dopo la caduta del fascismo sia INFAIL che INFPS hanno assunto le denominazioni conosciute oggi, ossia INAIL e INPS. Nel secondo dopoguerra nel 1952 si è adottata la legge che ha dato l'origine a trattenimento minimo di pensione. Negli anni '60 con lo scopo di tutelare i lavoratori che non potevano guadagnare non essendo responsabili di tale situazione è stata istituita la Cassa Integrazione Guadagni. Poi, negli anni successivi INPS sono entrate nel vigore alcune riforme ma si dice che il momento più importante nella trasformazione dell'ente a un'azienda moderna è la ristrutturazione del 1989. Nel detto anno è cominciata una serie di grandi modifiche della durata di circa 10 anni. In quel periodo fra l'altro sono state attuate le riforme relative agli altri tipi di lavoratori - autonomi e parasubordinati nonché è stata introdotta la previdenza complementare. La tutela dell'INAIL si è estesa e dal $2000^{5}$ comprende anche gli infortuni "in itinere" cioè durante il percorso da e verso il posto di lavoro. Inoltre nel 1992 è stata innalzata l'età pensionabile da 55 a 60 per le donne e da 60 a 65 per gli uomini ${ }^{6}$. In vista dell'aumento dell'aspettativa di vita previsto, negli ultimi anni è stata introdotta una nuova riforma che sta gradualmente elevando e equiparando l'età minima per la pensione di vecchiaia fino a 70 anni per tutti?

5 https://www.inail.it/cs/internet/istituto/chi-siamo/la-storia.html (accesso: 22.09.2017).

${ }^{6}$ https://www.inps.it/NuovoportaleINPS/default.aspx?itemdir=47209\&lang=IT (accesso: 22.09.2017).

7 http://www.borsaitaliana.it/notizie/speciali/pensioni/perche-la-riforma/eta-pensionabile/versolaparitatrauominiedonne.htm (accesso: 22.09.2017). 
La storia della previdenza sociale in Polonia è un po' più complicata. La Polonia non è esistita sulle carte geografiche per 123 anni a causa di spartizioni tra la Prussia, l'Impero Austriaco e l'Impero Russo. Prima di essi esistevano casse di mutuo soccorso però nel periodo in cui la previdenza sociale veniva codificata ed assumeva la sua forma, i territori polacchi facevano parte di tre altri paesi che sviluppavano in modo e tempo diverso. Solo due mesi dopo il recupero dell'indipendenza, nel gennaio di 1919 il capo dello Stato Józef Piłsudski ha emesso il decreto relativo all'assicurazione malattia ispirata al modello tedesco, però non l'ha passato al Parlamento. Per questo motivo gli storici dicono che la data ufficiale dell'inizio della previdenza sociale in Polonia è 19 maggio 1920 - il giorno dell'adozione della legge sull'assicurazione malattie obligatoria ${ }^{8}$. Grazie a tale normativa i lavoratori e le loro famiglie avevano la garanzia di ottenere l'assistenza sanitaria, ospedalizzazione e indennità di malattia quando ne avevano bisogno. Negli anni successivi sono entrate in vigore anche le disposizioni sull'assicurazione di disoccupazione, sull'assicurazioni uniforme di impiegati inoltre erano insaurate l'assicurazione malattia e l'assicurazione maternità nonché l'assicurazione in caso di inabilità lavorativa o di morte in seguito ad infortunio sul lavoro oppure malattia professionale. Tutte dette disposizioni furono concluse con il decreto presidenziale del $1934^{9}$ che univa cinque preesistenti indipendenti istituzioni creando ZUS - Ente di Previdenze Sociali.

Negli gli anni precedenti alla seconda guerra mondiale lo ZUS stava sviluppando velocemente. Tranne le attività come determinazione dei diritti a prestazioni ed erogazione di essi, gestione di previdenza pensionistica e di assicurazione contro i rischi di infortunio nonché trattamento e prevenzione delle malattie professionali si occupava della promozione di uno stile di vita sano e di sensibilizzazione sulla previdenza sociale. La gente non prendeva cura di sé ciò avrebbe potuto portare ad una morbilità superiore e la necessità di intervento da parte dello ZUS. Quindi venivano organizzate delle mostre itineranti con lo scopo di promuovere lo stile di vita sano. Si parlava di lotta contro l'alcol e contro le epidemie, di promozione dello sport nonché di come riposare bene dopo il lavoro. Nel secondo dopoguerra il governo socialista ha cominciato di interferire nel funzionamento dello ZUS. Nel 1955 ha anche soppresso questo ente. I suoi incarichi sono stati suddivisi tra le autorità locali e il Ministro del lavoro e della previdenza sociale. Tuttavia quel sistema non funzionava bene e solo cinque anni dopo l'amministrazione previdenziale è stata unificata in un solo istituto di ZUS come in precedenza. Negli anni successivi il sistema previdenziale subiva

${ }^{8}$ http://www.zus.pl/o-zus/o-nas/historia-zus/zus-80-lat-jak-jeden-dzien (accesso: 23.08 .2017$)$.

9 Ibidem. 
motli cambiamenti, da nazionalizzazione e instaurazione di monopolio di Stato, attraverso smantellamento di monopolio fino al mercato assicurativo libero introdotto grazie alla transizione del luglio del 1990. Il sistema si sta sempre evolvendo. La modificazione più controversa degli ultimi anni è, come in Italia, l'innalzamento dell'età pensionabile. Avrebbe stata elevata da 60 anni nel caso delle donne e 65 per quanto riguarda gli uomini al 67 per entrambi i sessi, però con l'inizio di ottobre del 2017 ha entrato in vigore una legge che farà tornare le cose allo stato precendente.

Come si può vedere, la storia aveva un grande impatto su come i sistemi amministrativi di diversi paesi si crearono. Il traduttore specializzato che si occupa delle assicurazioni sociali deve sapere come funzionano detti sistemi dei due stati che lo interessano nell'ambito del suo lavoro. Ogni paese ha un suo sistema indipendente che differisce dagli altri in qualche modo.

La prima cosa che contraddistingue il regime assicurativo polacco dall'italiano è la struttura. In Polonia esiste lo ZUS (Zakład Ubezpieczeń Społecznych - Ente di Previdenze Sociali) - un ente delle finanze pubbliche, invece in Italia, si può dire semplificando leggermente che le responsabilità dello ZUS polacco vengono divise su due enti pubblici non economici, [sottoposti alla sorveglianza del Ministero del Lavoro e delle Politiche Sociali] - INPS (Istituto Nazionale della Previdenza Sociale) e INAIL (Istituto Nazionale per l'Assicurazione contro gli Infortuni sul Lavoro).

All'INPS che viene definito come la più grande cassa previdenziale in Italia, sono assicurati quasi tutti i lavoratori dipendenti del settore privato, del settore pubblico, i lavoratori autonomi nonché i lavoratori precari. Gli incarichi più importanti dell'INPS consistono della retribuzione delle pensioni di natura previdenziale e di natura assistenziale. Le sue attività si possono dividere in quattro sezioni principali:

1) prestazioni previdenziali: ovviamente l'attività principale è quella previdenziale che consiste nell'erogazione delle pensioni e delle altre indennità. Tali indennità vengono calcolate in misura percentuale sulla retribuzione e finanziate dai contributi ricossi dai lavoratori. Le più comuni indennità di questo tipo sono: pensione di anzianità, pensione di vecchiaia, pensione di invalidità e pensione ai superstiti;

2) prestazioni a sostegno del reddito: hanno lo scopo di tutelare i lavoratori che hanno le difficoltà nella loro vita lavorativa e provvede aiuto finanziario alle persone con redditi modesti e famiglie numerose, ad esempio: indennità di malattia, indennità di maternità e congedo di paternità;

3) vigilanza: l'attività che controlla il versamento dei contributi in riferimento ad obblighi di legge, con lo scopo di affermare il rispetto delle norme e garantire giuste condizioni di concorrenza tra le imprese;

4) pubblico impiego. 
INAIL è un ente che eroga prestazioni ai lavoratori che si infortunano sul lavoro nonché quali che soffrono di malattie causate dall'attività lavorativa (chiamate le malattie professionali). Inoltre vengono tutelati i lavoratori che subiscono infortuni durante il viaggio di andata e ritorno dal luogo di lavoro. Gli obiettivi di quest'Istituto sono: riduzione del fenomeno infortunistico, garanzia del reinserimento nella vita sociale e lavorativa degli infortunati e degli affetti da malattia professionale nonché assicurazione dei lavoratori che svolgono attività a rischio.

Per poter denominare un evento infortunio, detto evento deve presentare certe caratteristiche: esteriorità, rapidità ed efficienza. Questo significa che l'incidente deve accadere al lavoratore a causa violenta cioè un fattore esterno che agisce con un'azione intensa e rapida e che causa una assoluta, ma non permanente, inabilità al lavoro per più di tre giorni. L'incidente che avviene durante il percorso verso il posto di lavoro o dal lavoro all'abitazione, e, qualora nell'azienda non sia presente un servizio di mensa, anche durante il percorso dal luogo di lavoro a quello dove il lavoratore consuma i pasti e viceversa, viene chiamato «infortunio in itinere». Il lavoratore che ha subito tale infortunio può non ottenere la prestazione in caso di inabilità al lavoro, quando durante l'infortunio si spostava utilizzando un mezzo privato, ad esempio l'automobile o la bicicletta, quando l'uso di questo non è necessario. L'utilizzo del mezzo privato può essere giustificato in alcune situazioni, tra le quali: quando il luogo di lavoro non è raggiungibile con i mezzi pubblici o quando la distanza dalla più vicina fermata dell'autobus o altro mezzo pubblico è eccesivamente lunga e non può essere percorsa in modo altro che a piedi.

Non si può dimenticare che INAIL, tranne il pagamento dell'indennità di infortunio, tutela i lavoratori anche in altri modi. Le altre competenze di questo ente consistono in, ad esempio: la rendita diretta per inabilità permanente; l'indennizzo per la menomazione dell'integrità psicofisica; l'assegno per assistenza personale continuativa; prestazioni protesiche, riabilitative o sanitarie; assegno funerario e prestazioni di reinserimento sociale e lavorativo.

Per quanto riguarda l'ente polacco, come è stato descritto di sopra, esso unisce quasi tutte le attività dell'INPS e dell'INAIL. Si occupa di erogazione delle indennità come indennità parentali, di malattia o pensioni, però fra tutte queste non c'è indennità di disoccupazione. Essa viene pagata da parte di un altro ente - Powiatowy Urząd Pracy (Ufficio Provinciale di Collocamento).

Le attività dello ZUS possono essere classificate quanto segue:

- pensioni: in questo gruppo c'è la pensione di vecchiaia e l'indennità di raccordo. Invece la pensione di anzianità, presente nel regime italiano, nel sistema polacco non esiste;

- rendite: cioè le indennità come pensione ai superstiti, la prestazione sociale nonché la pensione di invalidità e di inabilità; 
- indennità sostitutive della retribuzione: cioè le indennità di malattia dall'assicurazione contro le malattie, prestazione riabilitativa, assegno di assistenza, indennità compensativa, indennità di maternità e assegno funerario;

- prestazioni connesse a infortuni sul lavoro e a malattie professionali: a questo gruppo appartengono assegno di accompagnamento nonché la prestazione riabilitativa, l'indennità ai superstiti, di malattia, l'indennità compensativa, la pensione di invalidità e di inabilità menzionate prima che però in questo caso sono risultato di malattie professionali o infortuni sul lavoro.

Una diversa suddivisione di sistemi in Polonia e in Italia risulta in diverse indennità, diversa classificazione di esse e, di conseguenza, in terminologia, a volte, molto diversa. Nel sistema italiano ci sono delle indennità che non esistono nel regime polacco e vice versa, quindi un traduttore deve esprimere una cosa sconosciuta ad un dato sistema in un altro modo che sarebbe comprensibile a persone, a quali il sistema italiano e estraneo senza usare i termini già esistenti per non indurre in equivoco.

La prima questione potenzialmente problematica è collegata ai lemmi che rappresentano diversi tipi di erogazioni pagate dagli enti di assicurazione. Quasi in ogni caso la traduzione dipende dal contesto.

Il primo caso che va sottolineato è connesso al termine «indennità». In italiano questo lemma viene applicato riguardo alla maternità, alla malattia, alla mobilità, alla disoccupazione nonché ai pagamenti erogati alle persone pensionate o licenziate. In polacco, a seconda del contesto, la parola «indennità» può essere tradotta in quattro modi diversi. Quando si parla delle indennità di disoccupazione - erogata alle persone disoccupate che cercano lavoro, di malattia - versata alle persone che a causa di malattia non sono in grado di esercitare le proprie responsabilità professionali, oppure di maternità o di paternità - pagata ai genitori di un neonato bambino, nel regime polacco viene usata la voce $z a s i t e k$, rispettivamente: dla bezrobotnych, chorobowy e macierzyński o tacierzyński. Quando invece è il caso delle persone pensionate in un modo particolare, cioè parliamo di «indennità di raccordo», la parola giusta è emerytura (pomostowa), invece parlando delle persone licenziate e di «indennità di partenza»si deve usare il termine odprawa. E, alla fine, se ci riferiamo alla «indennità di immobilità» che in Polonia non esiste, bensì si utilizza il termine świadczenia mobilności.

La situazione è simile in caso del termine «pensione» che anche può essere tradotto in tre modi diversi: emerytura, renta o świadczenie. Nell'uso comune la parola «pensione» è tradotta come emerytura, però nel linguaggio specialistico viene tradotta così solo parlando della pensione di anzianità, ossia la prestazione che spetta dopo aver raggiunto una quota che consiste di somma tra l'età pensionabile minima richiesta (in Italia questa età è 60 per lavoratori dipendenti e 61 per lavoratori autonomi invece in Polonia è di 60 anni per le donne e 65 per gli uomini) e almeno 35 anni di contributi che in polacco viene tradotta come 
emerytura za wystugę. In riferimento alla pensione ai superstiti, alla pensione di invalidità civile o alla pensione sociale il lemma adeguato è renta, il quale viene tradotto in italiano anche come «rendita». L'ultima opzione e świadczenie che si applica in riferimento a: «la pensione di vecchiaia»- pagata alle persone che hanno raggiunto l'età pensionabile e hanno versato contributi per almeno 5 anni durante la vita lavorativa - in polacco świadczenie $z$ tytutu starości; «la pensione indennitaria» - erogata alle persone o alle famiglie di persone che hanno subito infortunio accaduto durante lo svolgimento dell'attività lavorativa che ha causato la menomazione o anche la morte - in polacco świadczenie odszkodowawcze o colloquialmente odszkodowanie nonché ai termini generali cioè «la pensione assistenziale» e «previdenziale» $\mathrm{i}$ quali vengono tradotti in polacco come świadczenie zdrowotne e społeczne.

Va notato che in italiano esistono due voci a dire bezrobotny. Il più comune è «disoccupato», però quando si parla di una persona senza occupazione che non l'ha mai avuta, in italiano viene usato termine «inoccupato».

Il «congedo» in polacco generalmente viene tradotto come urlop - wypoczynkowy, rodzicielski, macierzyński o ojcowski. Però c'è un'indennità in cui la voce «congedo» viene tradotta in un modo diverso - «congedo di malattia». In questo caso «congedo» si traduce come zwolnienie (lekarskie).

Per quanto riguarda la voce «assegno», la traduzione più frequente, però non unica, è zasiłek. In caso di «assegno di natalità» in polacco è usato il lemma świadczenie (porodowe) invece «assegno nel assegno per assistenza personale continuativa» viene tradotto come dodatek ( $z$ tytutu ciagtej pomocy osobistej). Per quanto riguarda assegni: familiare, funerario, sociale, di assistenza e di accompagnamento la traduzione giusta sarà zasitek. Si deve però aggiungere a questo punto qualcosa su «assegno di accompagnamento». Esso viene tradotto come zasitek pielegnacyjny, ma in polacco esiste anche indennità chiamata dodatek pielęgnacyjny. Nel linguaggio comune si sente usare zasitek e dodatek come sinonimi, ma si deve notare che in sistema polacco si parla di due indennità diverse.

L'ultimo caso di cui vorrei parlare riguarda i termini come wynagrodzenie, wypłata, pobory, uposażenie, płaca o pensja. In polacco la differenza più importante tra queste voci è che wynagrodzenie è più ampia e comprende anche prestazioni extrafinanziarie. Inoltre si può dire che pensja è un po' meno ufficiale invece uposażenie e pobory non sono usati molto frequentemente. Allora wynagrodzenie in italiano sarebbe «emolumenti» siccome questi due lemmi sono i più ampi e ufficiali. Invece per quanto riguarda voci come «remunerazione», «guadagno»e «retribuzione» si possono essere tradotti come placa, wyptata o pensja. In italiano ci sono anche lemmi riguardanti il pagamento che non si differiscono per il loro registro ma dal tipo di lavoro svolto da un lavoratore. Una persona che svolge un lavoro intellettuale ottiene lo «stipendio», invece un lavoratore manuale per il suo lavoro è remunerato con il «salario». In polacco non esiste una distinzione del genere. 
Tabella 1. Glossario italiano-polacco dei termini del campo di assicurazioni sociali

\begin{tabular}{|c|c|}
\hline ITALIANO & POLACCO \\
\hline $\begin{array}{l}\text { assegno } \\
\sim \text { di accompagnamento } \\
\sim \text { di assistenza } \\
\sim \text { di natalità } \\
\sim \text { familiare } \\
\sim \text { funerario } \\
\sim \text { per assistenza personale continuativa } \\
\sim \text { sociale }\end{array}$ & $\begin{array}{l}\text { zasiłek, świadczenie, dodatek } \\
\mathrm{z} \sim \text { pielęgnacyjny } \\
\mathrm{z} \sim \text { opiekuńczy } \\
\text { ś porodowe } \\
\mathrm{z} \sim \text { rodzinny } \\
\mathrm{z} \sim \text { pogrzebowy } \\
\text { dodatek z tytułu ciągłej pomocy osobistej } \\
\mathrm{z} \sim \text { socjalny }\end{array}$ \\
\hline $\begin{array}{l}\text { congedo } \\
\sim \text { annuale } \\
\sim \text { di malattia } \\
\sim \text { di maternità } \\
\sim \text { di paternità } \\
\sim \text { parentale }\end{array}$ & $\begin{array}{l}\text { urlop, zwolnienie } \\
\mathrm{u} \sim \text { wypoczynkowy } \\
\mathrm{z} \sim \text { lekarskie } \\
\mathrm{u} \sim \text { macierzyński } \\
\mathrm{u} \sim \text { ojcowski (pot. tacierzyński) } \\
\mathrm{u} \sim \text { rodzicielski }\end{array}$ \\
\hline $\begin{array}{l}\text { ferie/congedo } \\
\sim \text { non retribuite } \\
\sim \text { retribuite }\end{array}$ & $\begin{array}{l}\text { urlop } \\
\sim \text { bezpłatny } \\
\sim \text { wypoczynkowy }\end{array}$ \\
\hline $\begin{array}{l}\text { indennità } \\
\sim \text { di disoccupazione } \\
\sim \text { di frequenza } \\
\sim \text { di malattia } \\
\sim \text { di mancato preavviso } \\
\sim \text { di maternità } \\
\sim \text { di mobilità } \\
\sim \text { di partenza } \\
\sim \text { di raccordo } \\
\sim \text { compensativa }\end{array}$ & $\begin{array}{l}\text { emerytura, świadczenie, zasiłek, renta, } \\
\text { ekwiwalent/rekompensata } \\
\mathrm{z} \sim \text { dla bezrobotnych } \\
\mathrm{z} \sim \text { opiekuńczy dla osoby częściowo } \\
\text { niesamodzielnej } \\
\mathrm{z} \sim \text { chorobowy } \\
\text { r/e } \sim \text { z tytułu niezachowania ustawowego } \\
\text { okresu wypowiedzenia } \\
\text { z macierzyński } \\
\text { świadczenia mobilności } \\
\text { odprawa } \\
\text { e pomostowa } \\
\mathrm{z} \sim \text { wyrównawczy }\end{array}$ \\
\hline $\begin{array}{l}\text { pensione } \\
\sim \text { ai superstiti } \\
\sim \text { assistenziale } \\
\sim \text { di anzianità } \\
\sim \text { di inabilità } \\
\sim \text { di invalidità } \\
\sim \text { di invalidità civile }\end{array}$ & $\begin{array}{l}\text { emerytura, świadczenie, renta } \\
r \sim \text { rodzinna } \\
\text { ś } \sim \text { zdrowotne } \\
\text { e } \sim \text { za wysługę/stażowa } \\
r \sim \text { z tytułu całkowitej i trwałej niezdolności } \\
\text { do pracy } \\
\text { z } \sim \text { dla niepełnosprawnych } \\
\sim \text { renta }\end{array}$ \\
\hline
\end{tabular}


Tabella 1 (cont.)

\begin{tabular}{|l|l|}
\hline$\sim$ di reversibilità & $\mathrm{r} \sim$ rodzinna \\
$\sim$ di vecchiaia & ś z tytułu starości \\
$\sim$ indennitaria & ś odszkodowawcze (pot. odszkodowanie) \\
$\sim$ previdenziale & ś społeczne \\
$\sim$ privilegiata & $\begin{array}{l}\mathrm{r} \sim \text { uprzywilejowana [tytułem wypadku przy } \\
\text { pracy] } \\
\sim \text { sociale }\end{array}$ \\
\hline $\begin{array}{l}\text { disoccupato } \sim \text { socjalna } \\
\text { inoccupato }\end{array}$ & bezrobotny \\
\hline $\begin{array}{l}\text { disoccupazione } \\
\text { inoccupazione }\end{array}$ & bezrobocie \\
\hline $\begin{array}{l}\text { sussidio } \\
\sim \text { di assistenza }\end{array}$ & zasiłek \\
$\sim$ di disoccupazione & $\sim$ opiekuńczy \\
\hline
\end{tabular}

\section{Bibliografia}

Cinelli M., Diritto della previdenza sociale, Giapichelli, Torino 2015, cap. II.

Orlicki M., Ubezpieczenia obowiązkowe, Wolters Kluwer, Warszawa 2011, cap. I.

System ubezpieczeń społecznych. Zagadnienia podstawowe, a cura di G. Szpor, Wolters Kluwer, Warszawa 2016.

Szczęśniak M., Zarys dziejów ubezpieczeń na ziemiach polskich, Przedsiębiorstwo Wydawnicze LAM, Warszawa 2003, cap. I e XI.

\section{Sitografia}

http://www.borsaitaliana.it/notizie/speciali/pensioni/perche-la-riforma/etapensionabile/versolaparitatrauominiedonne.htm (accesso: 22.09.2017).

http://www.storiadellasicurezzasociale.ch/fileadmin/synthese-it.pdf (accesso: 22.09.2017).

http://www.zus.com.pl/files/prasa/Pocz\%C4\%85tki\%20i\%20rozw\%C3\%B3j\%20 ubezpiecze\%C5\%84.pdf (accesso: 22.09.2017).

http://www.zus.pl/o-zus/o-nas/historia-zus/zus-80-lat-jak-jeden-dzien (accesso: 23.09.2017).

https://www.inail.it/cs/internet/istituto/chi-siamo/la-storia.html (accesso: 22.09.2017).

https://www.inps.it/NuovoportaleINPS/default.aspx?itemdir=47209\&lang=IT (accesso: 22.09.2017). 